\title{
Top Quark Mass from the Tevatron and LHC Colliders
}

\author{
Luca Brigliadori ${ }^{1, a}$ \\ ${ }^{1}$ Dipartimento di Fisica dell' Università di Bologna \\ Viale Berti-Pichat 6/2, 40127 - Bologna, Italy
}

\begin{abstract}
The discovery of the top quark in 1995 has been one of the great successes of the CDF and D0 experiments at the Fermilab Tevatron collider. Since then, many measurements of the top quark properties have been performed in different channels and using many methods. The importance of measuring its mass lies in the possibility of verifying the predictions and the consistency of the Standard Model as well as in setting constraints on possible, still unobserved, physics. In 2010, the new CERN experiments, ATLAS and CMS, started to measure the top quark properties exploiting the large amount of data collected at the Large Hadron Collider. In March 2014, the very first combination of measurements from all the four experiments has been performed yielding $\mathrm{M}_{\text {top }}=173.34 \pm 0.76 \mathrm{GeV}$, with a precision below $0.5 \%$. In these proceedings a selected review of the most recent or relevant results obtained by the Tevatron and LHC Collaborations is presented.
\end{abstract}

\section{Introduction}

Since the first observation of the top quark ( )tby the CDF and D0 experiments at the Tevatron collider in $1995[1,2]$ the large value of its mass $\left(\mathrm{M}_{\mathrm{top}}\right)$ represents a striking property of this particle. If the scalar boson observed by the ATLAS and CMS experiments at the CERN Large Hadron Collider (LHC) [3] is identified as the Standard Model (SM) Higgs boson, the top quark is by far the heaviest particle in the SM with the three generations of fermions observed so far. This makes its contribution to higher order corrections to many electroweak observables dominant so that a precise knowledge of $\mathrm{M}_{\text {top }}$ is fundamental in checking the consistency of theoretical predictions of the SM by the electroweak fits [4]. This and other reasons pushed the CDF and D0 collaborations to measure $\mathrm{M}_{\text {top }}$ in all possible topologies related to top quark pair $(t \bar{t})$ events production, and to improve the used techniques. Shortly after the Tevatron shutdown in September 2011, the whole samples of data collected by the experiments, corresponding to about $10 \mathrm{fb}^{-1}$ of $p \bar{p}$ collisions each, became available for analyses, allowing therefore to reach the smallest achievable statistical uncertainty.

Since 2010, also the new experiments at the LHC, ATLAS and CMS, started to take data and consequently to perform measurements concerning the top quark properties, including obviously its mass. The large $t \bar{t}$ production cross section and the high istantaneous luminosity of the collider allowed to collect large amount of data from $p p$ collisions both in the 2011 Run (about $5 \mathrm{fb}^{-1}$ per experiment) and especially in the 2012 Run (about $20 \mathrm{fb}^{-1}$ per experiment) so that results with a very good precision have been obtained in a short time.

\footnotetext{
ae-mail: brigliad@bo.infn.it
}

\section{Top Quark Production, Decay and Signatures}

In the Run II of the Tevatron, from March 2001 to September 2011, bunches of protons and antiprotons were brought into collision with a center-of-mass energy, $\sqrt{s}$, equal to $1.96 \mathrm{TeV}$ and data were collected by the multipurpose CDF and D0 detectors [5, 6]. At this energy, top quarks are predominantly produced in $t \bar{t}$ pairs by $q \bar{q}$ annihilation ( $\sim 85 \%$ of the times) or gluon-gluon fusion $(\sim 15 \%)$ with a total cross section in the range $7.0 \div 7.6 \mathrm{pb}$ for $\mathrm{M}_{\text {top }}=172.5 \mathrm{GeV}$ [7].

The pair production is the dominant process also in the proton-proton collisions at the LHC. Here the centerof-mass energy of the proton bunches amounted to 7 and $8 \mathrm{TeV}$ in 2011 and 2012, respectively, and the resulting events were recorded by the ATLAS and CMS detectors, described in details in [8,9]. Given the larger energies of the colliding beams the $t \bar{t}$ production cross section is increased with respect to the Tevatron, with values close to $170 \mathrm{pb}$ at $\sqrt{s}=7 \mathrm{TeV}$ and $250 \mathrm{pb}$ at $\sqrt{s}=8 \mathrm{TeV}$ [10], with the dominant contribution $(\sim 90 \%)$ from the gluongluon fusion mechanism.

In the SM framework the top quark decays to a $W$ boson and a $b$-quark with a branching ratio (BR) very close to $100 \%$ so that the different final states of $t \bar{t}$ ("signal") events can usually be classified based on the decays of the $W$ boson pair to leptons $\left(W \rightarrow \ell v_{\ell}\right)$ or quarks $\left(W \rightarrow q_{1} \bar{q}_{2}\right)$ as the "di-lepton channel" $(\mathrm{BR} \simeq 9 \%)$, the "lepton + jets channel" $(\mathrm{BR} \simeq 45 \%)$ and the "all-hadronic channel" (or all-jets channel, $\mathrm{BR} \simeq 46 \%$ ). The signal signatures used in analyzing the data correspond to the final states and have similar nomenclature, but usually the "di-lepton" and "lepton + jets" analyses consider only events with elec- 
trons and muons directly produced from the $W$ decays, excluding therefore events with $\tau$ leptons and reducing the BR's to about $5 \%$ and $30 \%$, respectively. In order to cover all the possible kind of events a "missing transverse energy + jets" $\left(H_{T}+\right.$ jets $)$ signature is also defined.

The ratio of $t \bar{t}$ and total cross sections is such that both at Tevatron and LHC one signal event is produced in about $10^{10}$ inelastic $p \bar{p}$ or $p p$ collisions. This makes the measurement of any of the top quark properties a really challenging task, requiring tools and selection techniques exploiting at the best the peculiar features of the signal. An important example is the identification of jets generated by $b$-quarks (" $b$-tagging"), fundamental in reducing the background yield and also the combinatoric problem related to possible jet-to-quark assignments. In measuring $\mathrm{M}_{\text {top }}$, the reconstruction of the kinematic properties of the event is crucial. The estimate of the parton energy requires an accurate knowledge of the correction to be applied to the measured jet energy (Jet Energy Scale, JES, or Jet Scale Factor, JSF), due to instrumental effects or jet clustering algorithms.

\section{Measurement Techniques}

Apart from the peculiarities of each individual measurement and with a few exceptions, two main techniques are used to extract the value of $\mathrm{M}_{\text {top }}$ from a sample of selected events : the Matrix Element Method (ME) and the Template Method (TMT).

In the ME, the probability that a set $\vec{y}$ of variables is observed is evaluated as a function of the possible event kinematics $\vec{x}$ (depending on $\mathrm{M}_{\text {top }}$ for $t \bar{t}$ ) at parton level, given "transfer functions" $\mathcal{W}(\vec{y}, \vec{x})$ taking into account detector effects and the event reconstruction. In the TMT a set of event observables, $\vec{y}$, sensitive to $\mathrm{M}_{\text {top }}$ is considered and their expected distributions are used as references ("templates") for the data in the measurement. In both methods a likelihood, written as the product of individual event probabilities, is then usually maximized as a function of $\mathrm{M}_{\text {top }}$ to extract the value which gives the largest probability to observe the selected set of events.

A well established and important feature of most analyses is the calibration of the JES simultaneously (in situ) with the $\mathrm{M}_{\text {top }}$ measurement, constraining the fourmomenta of jets assigned to a $W$ by its well known mass. This technique allows a part of the JES uncertainty to scale down with the amount of data collected.

\section{Results of $M_{t o p}$ Measurements}

In this section a brief summary of most recent results obtained by the Tevatron and/or LHC Collaborations regarding $\mathrm{M}_{\text {top }}$ is presented. A more complete overview can be found in [11].

The Tevatron Collaborations are updating all the analyses to include the full set of data collected by the detectors during the Tevatron Run II. This task has been completed for all channels by the CDF experiment, where all the new measurements are based on TMT, and it is in progress by D0, that anyway already obtained the most precise single measurement in the world, so far.

As it concerns the LHC experiments, some results have been obtained using data at $\sqrt{s}=8 \mathrm{TeV}$, while the remaining are still based on the 2011 data samples.

For all the measurements the available integrated luminosity is a function of the main triggers and subdetectors involved in the event selection and reconstruction.

\subsection{Di-Lepton Channel}

The di-lepton or fully leptonic channel usually provides the candidate samples with the best signal-to-background ratio $(S / B)$ because of the presence of two energetic, high$p_{\mathrm{T}}$ leptons and the $b$-jets. Moreover, the combinatoric problem in assigning jets to partons is small. Unfortunately, it suffers of a small BR (Sec. 2) and the kinematics of the events is underconstrained because the reconstructed $H_{T}$ results from two undetected neutrinos. As there is no $W$ decaying to hadrons, the in situ JES calibration cannot be performed in this channel.

The most precise measurements in this channel come from analyses performed by the LHC Collaborations, both exploiting the TMT method on about $5.0 \mathrm{fb}^{-1}$ of data at $\sqrt{s}=7 \mathrm{TeV}[12,13]$.

The result by ATLAS Collaboration is obtained with an integrated luminosity of $4.7 \mathrm{fb}^{-1}$ where a total of 2913 events are selected in the $e^{+} e^{-}, e^{ \pm} \mu^{\mp}$ and $\mu^{+} \mu^{-}$ channels by requiring the presence of exactly two oppositely charged energetic leptons, missing transverse energy and at least two energetic hadronic jets, with exactly two of them tagged as $b$-jets. For these events, a variable $\left(m_{\ell b}\right)$ based on invariant masses of the two lepton-tagged jet pairs is reconstructed and compared by a likelihood fit to the templates expected from simulations as a function of $\mathrm{M}_{\text {top }}$. Figure 1 just shows the expected distributions for simulated signal events corresponding to different values of $\mathrm{M}_{\mathrm{top}}$. The result of the fit gives $\mathrm{M}_{\text {top }}=173.09 \pm 0.64$ (stat) \pm 1.50 (syst) GeV , corresponding to a relative uncertainty $\approx 0.94 \%$.

A similar event selection, at least as it concerns the fundamental requirements, is performed in the CMS analysis [13], but here all events with at least one tagged jets are considered. An "Analytical Matrix Weighting Technique" (AMWT) is used to reconstruct a top quark mass, $m_{\mathrm{AMwT}}$, event by event. This consists in assuming a value $m_{t}$ of the mass and obtaining the corresponding multiple solutions for the undetected momenta of neutrinos. Each solution is given a weight related to the probability density of the event observed kinematics and finally the value of $m_{t}$ with the largest sum of weights of the corresponding solutions is taken as the $m_{\text {AMwT }}$ of the event. Distributions of $m_{\text {AMwT }}$ are built by simulated events with different values of the input $\mathrm{M}_{\text {top }}$ and used as templates in the measurement that gives $\mathrm{M}_{\text {top }}=172.5 \pm 0.4$ (stat) \pm 1.5 (syst) GeV, i.e. a precision $\approx 0.93 \%$. The plot of Fig. 2 shows the observed $m_{\mathrm{AMWT}}$ distribution compared to what is expected for $\mathrm{M}_{\text {top }}=172.5 \mathrm{GeV}$ together with the behavior of the likelihood function around the fitted value of the top quark mass. 


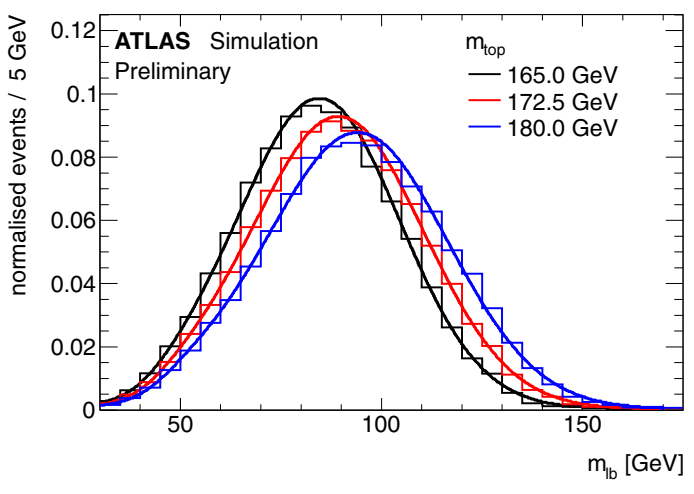

Figure 1. Dependence on the input top quark mass $\mathrm{M}_{\text {top }}$ of the distributions of the variable $m_{\ell b}$ used as template in the ATLAS analysis [12].

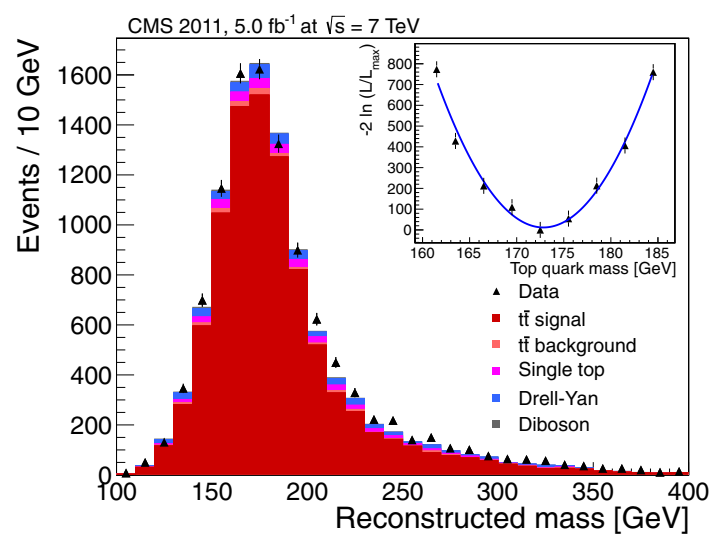

Figure 2. Distribution of the reconstructed mass in data and simulation for a top quark mass hypothesis of $172.5 \mathrm{GeV}$ with the AMWT method used in [13]. The inset shows the behavior of the likelihood close to the fitted value, corresponding to the minimum of $-2 \ln \left(\mathcal{L} / \mathcal{L}_{\max }\right)$.

The updated TMT analysis from the CDF Collaboration [14] is based on $9.1 \mathrm{fb}^{-1}$ of data and represents the most recent result in this channel. Two different event variables are considered : a top quark mass $\left(\mathrm{M}_{t}^{\mathrm{reco}}\right)$, reconstructed by the so-called "Neutrino Weighting Algorithm" (NWA), and an "alternative" mass $\left(\mathrm{M}_{t}^{\text {alt }}\right)$ which is not based on the energies of jets to be insensitive to the JES, so that its distribution is not affected by the corresponding uncertainty. An hybrid variable $\left(\mathbf{M}_{t}^{\mathrm{eff}}\right)$, obtained as a weighted sum of $\mathrm{M}_{t}^{\text {reco }}$ and $\mathrm{M}_{t}^{\text {alt }}$, is then defined to build the template distributions used in the measurement. The likelihood fit to the data gives $\mathrm{M}_{\text {top }}=170.80 \pm 3.25 \mathrm{GeV}$, with a total relative precision of $\approx 1.9 \%$. Figure 3 shows the fitted templates for events with no $b$-tagged jet.

Two different results are combined by the D0 Collaboration to obtain a better measurement in this channel. The result of a ME analysis performed on $5.4 \mathrm{fb}^{-1}$ of data [15] is combined with one using TMT with NWA [16] and based on a total of $5.3 \mathrm{fb}^{-1}$. An example of templates used in the latter analysis is shown in Fig. 4. A peculiarity of this analysis is that the JES is calibrated using a value ob-

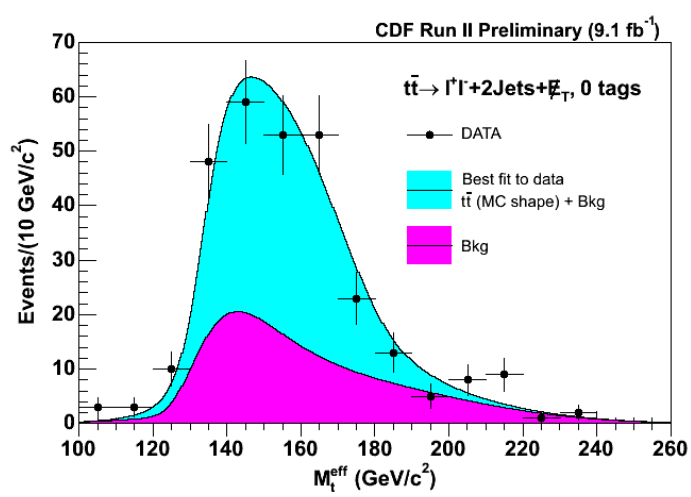

Figure 3. Observed data and fitted templates for events with no $b$-tagged jet in the $\mathrm{CDF} \mathrm{M}_{\mathrm{top}}$ measurement in the di-lepton channel [14].

tained in the lepton + jets channel [17]. The final result is $\mathrm{M}_{\mathrm{top}}=173.9 \pm 2.4 \mathrm{GeV}$, with a precision of $\approx 1.4 \%$.
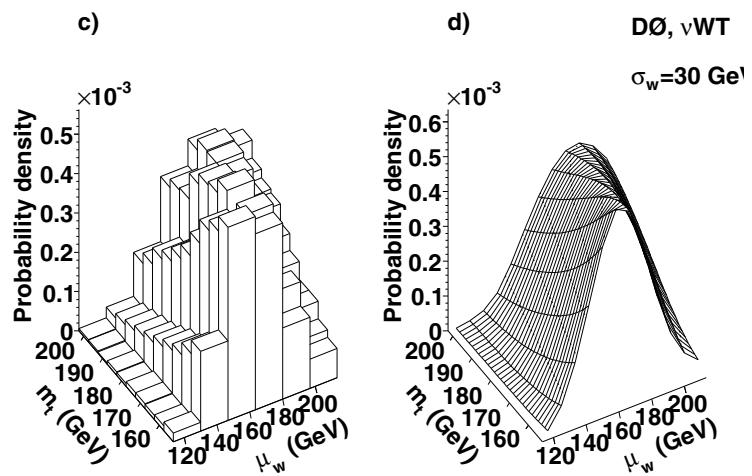

Figure 4. Example of templates (both histogram, left, and the corresponding smooth parametrization, right) as a function of the input top quark mass as used in the di-lepton measurement by D0 [16].

\subsection{Lepton + Jets Channel}

The final state including one charged lepton and jets can be considered the "golden channel" as it concerns the measurements of the top quark properties. In fact it offers the best compromise between the purity of selected samples and their amount of data because of its large BR. This allows all the experiments to achieve their best results in this channel and, consequently, these include the most precise single measurements of $\mathrm{M}_{\text {top }}$ in the world. At present, the latter are represented by two analyses performed by the D0 and CMS Collaborations, achieving a precision close to $0.4 \%$ with absolute uncertainties $\approx 0.8 \mathrm{GeV}$.

A recent result by the D0 experiment [18], based on a ME method analysis, achieves the smallest absolute uncertainty in a single measurement so far, using the full data sample collected by the detector $\left(9.7 \mathrm{fb}^{-1}\right)$. This has been possible through an update of the detector calibrations and the improvement of the Monte Carlo simulation of $t \bar{t}$ signal. Events are selected with one isolated and energetic 
electron or muon and four high tranverse momentum $\left(p_{\mathrm{T}}\right)$ jets at least one of which must be $b$-tagged. An event probability is then defined using simulated events as a function of $\mathrm{M}_{\text {top }}$ and, through the transfer functions, of the JES, taking into account the agreement between the invariant mass of jets assigned to the $W$ boson and the nominal $W$ mass. Maximizing the total probability for the observed data, $\mathrm{M}_{\text {top }}=174.98 \pm 0.41$ (stat) \pm 0.64 (syst) GeV is obtained, where the systematic uncertainty here includes also the statistical contribution related to the in situ JES calibration, with a total precision $\approx 0.43 \%$. Figure 5 shows, for the invariant mass of jets assigned to one of the $W$ bosons, the agreement between the observed data and the expected distribution corresponding to the measured values of $\mathrm{M}_{\text {top }}$ and JES.

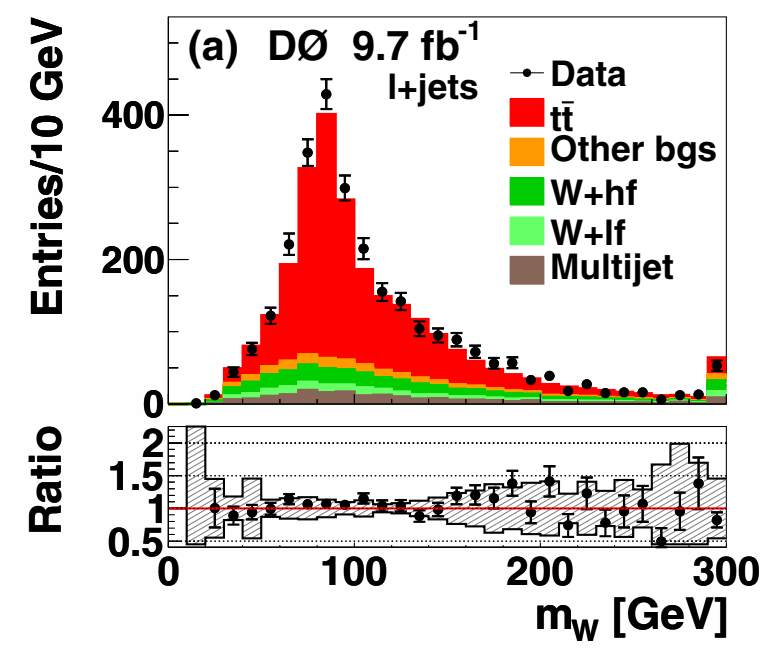

Figure 5. Invariant mass of the jet pair assigned to one of the $W$ bosons for data and expected distributions corresponding to the measurements obtained in [18]. In the ratio of observed data to expectation from simulated events, the total systematic uncertainty is shown as a shaded band.

The last measurement performed by the CMS Collaboration in this channel [19] is based on $19.7 \mathrm{fb}^{-1}$ of data collected during the 2012 Run of the LHC at $\sqrt{s}=8 \mathrm{TeV}$ and uses the so-called "ideogram method" where templates are used to evaluate a likelihood function for the observed data. The event selection requires exactly one energetic lepton and at least four jets with exactly two $b$-tags in the four ones with the largest $p_{\mathrm{T}}$. Moreover, the requirement of a good convergence of the fit used to reconstruct the event kinematics is applied. The distribution of the top quark mass $m_{t}^{\mathrm{fit}}$, as reconstructed for each possible assignment of the observed lepton and jets to the partons of a $t \bar{t}$ final state and weighted by its fit probability, is used as the main template, while the invariant mass of jets assigned to the $W$ boson in the fit, $m_{W}^{\text {reco }}$, is used as the reference distribution for the in situ calibration of the JES, given its sensitivity to this parameter and, on the contrary, the independence on $\mathrm{M}_{\text {top }}$. To improve the statistical power of the method, templates are derived separately from simulated events in case of right and wrong assignments of jets to partons. An example of expected distributions for $m_{t}^{\text {fit }}$ is shown in Fig. 6.

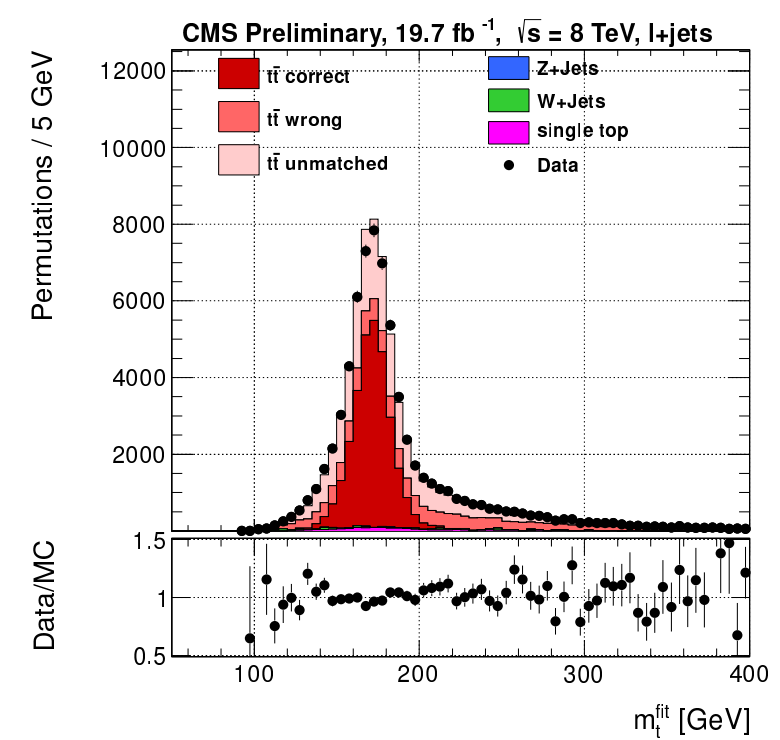

Figure 6. Expected distribution of the variable $m_{t}^{\text {fit }}$ used as main template in [19] compared to observed data. For the simulated signal events " $t \bar{t}$ correct", " $t \bar{t}$ wrong" and " $t \bar{t}$ unmatched" refer to events with the right, wrong and undefined assignment of observed jets to the partons of the lepton plus jets final state, respectively.

The likelihood fit to the total of 28750 events in the data, performed as a function of $\mathrm{M}_{\text {top }}$ and JES gives $\mathrm{M}_{\text {top }}=172.04 \pm 0.19$ (stat $\left.+\mathrm{JES}\right) \pm 0.75$ (syst) GeV, where the JES uncertainty is joined to the statistical contribution because both arise directly from the fit.

Two more recent measurements, both based on TMT and performed by the ATLAS and CDF Collaborations, respectively, can be considered.

The result obtained by ATLAS [20] uses $4.7 \mathrm{fb}^{-1}$ of data at $\sqrt{s}=7 \mathrm{TeV}$. This measurement has the peculiarity to be the first one where a further reduction of the systematic contribution of the JES is obtained by using a 3-dimensional template fit where, beside the usual event reconstructed top quark and $W$ masses $\left(m_{t}^{\text {reco }}\right.$ and $m_{W}^{\text {reco }}$, respectively), a third distribution, $R_{\ell b}^{\text {reco }}$, built from ratios of the $p_{\mathrm{T}}$ 's of tagged and untagged jets is used to exploit its sensitivity to the possible differences in the JES for $b$ and light flavor jets and in particular to their ratio, denoted as $b \mathrm{JSF}$. An example of this distribution is shown in Fig. 7. This is done to obtain an in situ calibration of both the scales and allows a reduction of about $24 \%$ of the total uncertainty on $\mathrm{M}_{\text {top }}$ with respect to the same analysis when performed using the usual 2-dimensional approach. The measurement gives $\mathrm{M}_{\text {top }}=172.31 \pm 0.75$ (stat $\left.+\mathrm{JSF}+b \mathrm{JSF}\right) \pm 1.35$ (syst) GeV , with a relative precision $\approx 0.9 \%$.

Also the measurement by the CDF Collaboration [21] is performed by a 3-dimensional template method, but without adding any further parameter to the likelihood fit, so that the improvement with respect a 2dimensional result concerns above all the statistical 


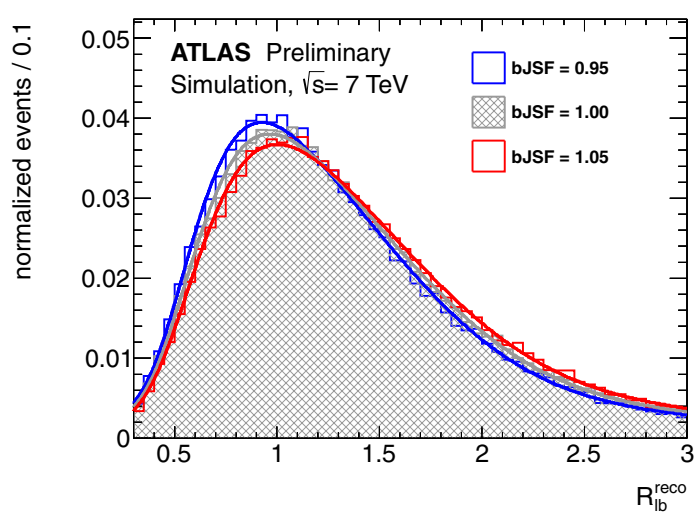

Figure 7. Distribution of the $R_{\ell b}^{\text {reco }}$ variable used in [20] and its dependence on a relative scale $b \mathrm{JSF}$ between the jet energy scales of $b$ and light flavor jets. The example refers to events with at least two $b$-tagged jets.

power. As usual the invariant mass of jets assigned to the $W$ boson in the event kinematics reconstruction is used to exploit the JES calibration, while two different top quark masses $\left(m_{t}^{\text {reco }}\right.$ and $\left.m_{t}^{\text {reco (2) }}\right)$ are considered for deriving the two more reference distributions. The result, obtained with $8.7 \mathrm{fb}^{-1}$ of data, is $\mathrm{M}_{\text {top }}=172.85 \pm 0.71$ (stat $\left.+\mathrm{JES}\right) \pm 0.85$ (syst) GeV.

\subsection{All-Hadronic Channel}

The all-hadronic channel has the advantage of the large $\mathrm{BR}$ and the possibility to reconstruct completely the kinematic properties of the event because ideally no particle from the $t \bar{t}$ system escapes the detector. The major downside is the huge background from QCD multijet production which usually greatly dominates the signal even after the application of specific triggers. Probably due to the difficulties to obtain a small precision and a good estimate of the QCD background, only a few measurements exist in this channel so far, and the recent ones have been performed by the CMS, ATLAS and CDF Collaborations using the TMT method.

The CMS analysis, described in [22], is performed using the same method and variables of the lepton plus jets measurement [19] and similar requirements in the event selection as it concerns the goodness of event reconstruction, apart from obvious differences related to the two channels. Therefore a high energetic jets multiplicity and exactly two $b$-tags are required to match the number and flavor of quarks in the fully hadronic $t \bar{t}$ final state, with the two tagged jets at a distance $\Delta R_{b \bar{b}}>0.2$ in the $\eta-\phi$ space. The event selection allows to reach a very good expected purity $(\approx 78 \%$ ) of the sample of 4356 candidate $t \bar{t}$ events. Figure 8 shows, for $\mathrm{M}_{\text {top }}=172.5 \mathrm{GeV}$ and the default JES value, the expected distribution of the reconstructed top quark mass compared to data.

The maximization of the likelihood given the observed data yields $\mathrm{M}_{\text {top }}=172.08 \pm 0.36$ (stat $\left.+\mathrm{JSF}\right) \pm 0.83$ (syst) GeV,

with a relative precision $\approx 0.53 \%$, very close to what

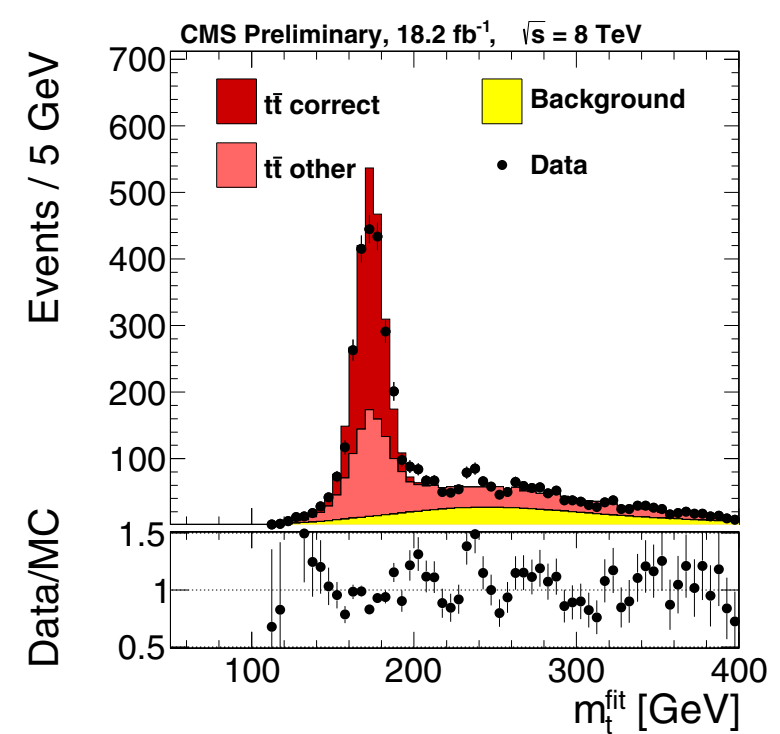

Figure 8. Expected distributions, for $\mathrm{M}_{\mathrm{top}}=172.5 \mathrm{GeV}$ and default jet energy scale, for the variable $m_{t}^{\text {fit }}$ used as main template in [22] compared to observed data. For the simulated signal events " $t \bar{t}$ correct" and " $t \bar{t}$ other" refer to events with the right and wrong or undefined assignment of hadronic jets to the partons of the all-adronic final state, respectively.

obtained in the lepton plus jets channel (Sec. 4.2) which suffers of much lower background.

A result in this channel, based on $4.6 \mathrm{fb}^{-1}$ of data collected in 2011, has been recently obtained also by the ATLAS Collaboration [23]. A TMT measurement is applied after the event selection requiring at least six high $p_{\mathrm{T}}$ hadronic jets with exactly two $b$-tags among the four leading ones, where the ratio $R_{3 / 2}$ of the invariant mass $m_{j j j}$ of the three jets assigned to a top quark in the event reconstruction to the one of the two jets assigned to the corresponding $W$ boson, $m_{j j}$, is used as the variable to build the templates. In this analysis no in situ JES calibration is performed, while a cancellation of systematic effects common to both $m_{j j j}$ and $m_{j j}$ (like the JES contributions) is achieved by using their ratio $R_{3 / 2}$. A binned likelihood fit to the data gives $\mathrm{M}_{\text {top }}=175.06 \pm 1.35$ (stat) \pm 1.22 (syst) GeV, i.e. a relative uncertainty $\approx 1.0 \%$.

The most recent analysis by the CDF Collaboration [24] is based on $9.3 \mathrm{fb}^{-1}$ of data selected by a multijet trigger. An event selection exploiting a Neural Network with 13 input kinematic and jet shapes variables is used, together with the requirement of at least one $b$-tagged jet, to obtain a good signal-to-background ratio in this difficult channel. Two variables, a "top quark mass" $m_{t}^{\text {reco }}$ and a " $W$ mass" $m_{W}^{\text {reco }}$, are reconstructed event by event using kinematic fits and their distributions are used as templates. The result from the likelihood fit, including JES calibration, is $\mathrm{M}_{\text {top }}=175.07 \pm 1.19$ (stat) \pm 1.56 (syst) GeV, with a precision $\approx 1.1 \%$. Figure 9 shows the dependence of $m_{W}^{\text {reco }}$ distribution on JES for an input $\mathrm{M}_{\mathrm{top}}=172.5 \mathrm{GeV}$. 


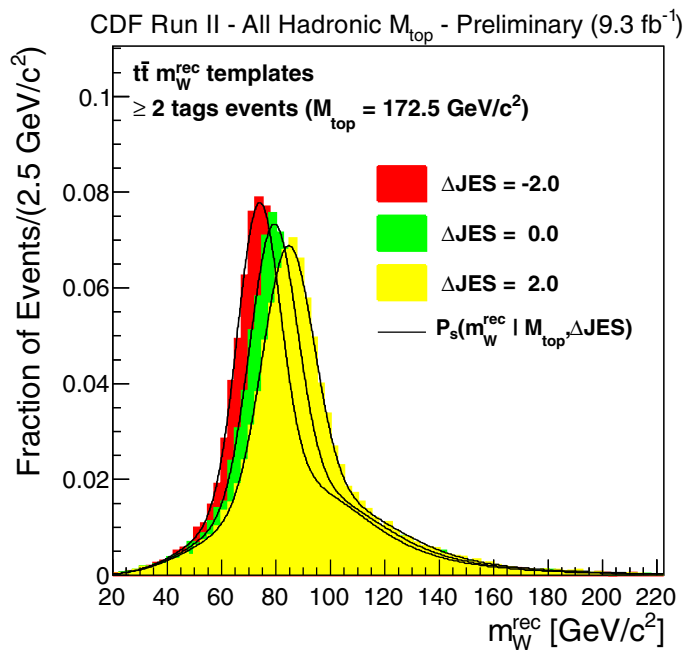

Figure 9. Distributions of the reconstructed $W$ mass, $m_{W}^{\text {reco }}$, as a function of the input jet energy scale for simulated events with at least $2 b$-tagged jets selected in the analysis [24] for an input top quark mass of $172.5 \mathrm{GeV}$. The parameter $\triangle \mathrm{JES}$ denotes the displacement of JES from the default central value using the uncertainty on the latter as the unit. The corresponding parametrizations by smooth functions are also shown.

\section{4 $B_{T}+$ jets Channel}

As outlined in Sec. 2 the $H_{T}+$ jets signature is usually defined to be complementary to all the other measurement channels, with large $\mathbb{H}_{T}$ (differently from the all-hadronic channel) and no energetic and isolated lepton (to reject events falling in the usual leptonic channels) required in order to obtain a statistically independent data sample.

In this channel, a result obtained by CDF with the full dataset $\left(8.7 \mathrm{fb}^{-1}\right)$ is available [25]. The analysis presents features similar to both the all-hadronic [24], as it concerns the background modeling and kinematical event selection, and to the lepton + jets [21] one, as the same variables are reconstructed for the templates. In the event reconstruction the $B_{T}$ is totally assigned to the $W$ boson decaying leptonically, assuming that also the charged lepton escapes detection. The result is $\mathrm{M}_{\text {top }}=173.93 \pm 1.26$ (stat) \pm 1.36 (syst) $\mathrm{GeV}$ yielding a precision of $1.1 \%$. Figure 10 shows the expected distribution of the output of the Neural Net used in the event selection compared to data.

\subsection{Indirect $\mathrm{M}_{\text {top }}$ measurements from the $t \bar{t}$ cross section}

In Quantum Chromodynamics (QCD) the masses of quarks are not observable quantities as quarks are confined inside hadrons. Therefore the mass parameters in the QCD lagrangian depend on the renormalization scheme used to define the theory [26]. Given the precision reached by the current results, it is important to define the meaning of the $\mathrm{M}_{\text {top }}$ parameter obtained by the direct measurements at the high energy colliders. As all the latter rely on Monte Carlo simulations of the $t \bar{t}$ production process, that parameter corresponds to what implemented in the event genera-

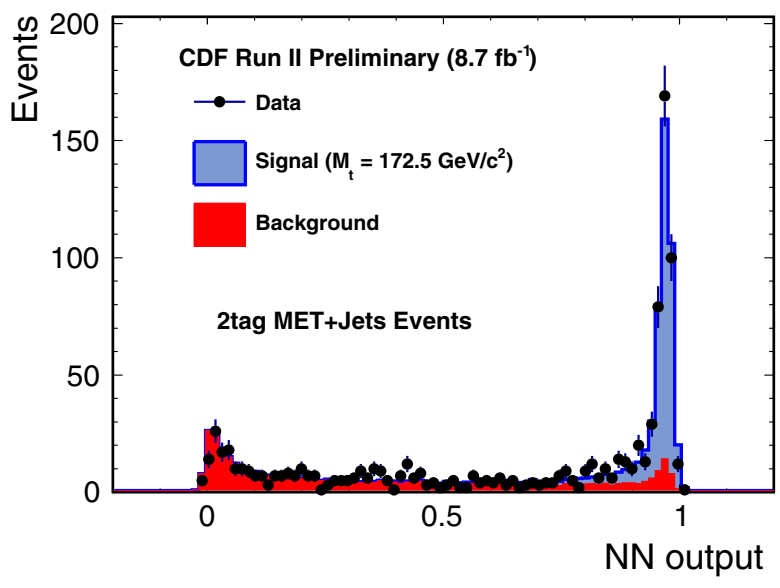

Figure 10. The distribution of the output of the Neural Net used in analysis [25] for events with two $b$-tagged jets is shown, before the final requirement ( $\mathrm{NN}$ output $\geq 0.8$ ) on the output itself, for data and simulated signal and background.

tors and should be close (within $\sim 1 \mathrm{GeV}$ ) to the top quark "pole mass" [27], which, anyway, has an intrinsic uncertainty of order $\Lambda_{\mathrm{QCD}}$ [28]. Indirect measurements of $\mathrm{M}_{\mathrm{top}}$ through comparison of the measured $t \bar{t}$ cross section to its theoretical prediction as a function of the top quark mass itself allow to obtain a result within a well defined renormalization scheme.

The most recent and precise measurement using this technique has been performed by the CMS Collaboration [29]. This is based on a measurement of the $t \bar{t}$ cross section obtained with $2.3 \mathrm{fb}^{-1}$ of data collected during the 2011 run of the LHC at $\sqrt{s}=7 \mathrm{TeV}$ in the di-lepton channel and a next-to-next-to-leading order (NNLO) calculation including next-to-next-to-leading logarithms (NNLL). The quantity $\mathrm{M}_{\text {top }}$ is extracted as the value maximizing the probability to obtain the observed value and the theoretical prediction simultaneously, taking into account their respective uncertainties :

$$
P\left(\mathrm{~m}_{t}\right)=\int f_{\exp }\left(\sigma_{t \bar{t}} \mid \mathrm{m}_{t}\right) \cdot f_{\mathrm{th}}\left(\sigma_{t \bar{t}} \mid \mathrm{m}_{t}\right) \mathrm{d} \sigma_{t \bar{t}}
$$

where $f_{\text {exp }}$ and $f_{\text {th }}$ are the probability density functions of the measured and calculated cross section, respectively. The measurement, obtained for the top quark pole mass, is $\mathrm{M}_{\mathrm{top}}^{\text {pole }}=176.7_{-2.8}^{+3.0} \mathrm{GeV}$, in agreement with the direct measurements. Figure 11 shows the behavior of both the theoretical and measured cross sections as a function of the pole $\mathrm{M}_{\text {top }}$ and the $P\left(\mathrm{~m}_{t}\right)$ function used to extract the result.

\section{$5 \mathbf{M}_{\text {top }}$ measurements combinations}

The Tevatron and LHC Collaborations regularly combine their best results both internally (i.e. within each experiment separately) and between experiments at the same collider to obtain values representing the Tevatron and LHC $\mathrm{M}_{\text {top }}$ averages. Moreover, in March 2014, the very first 

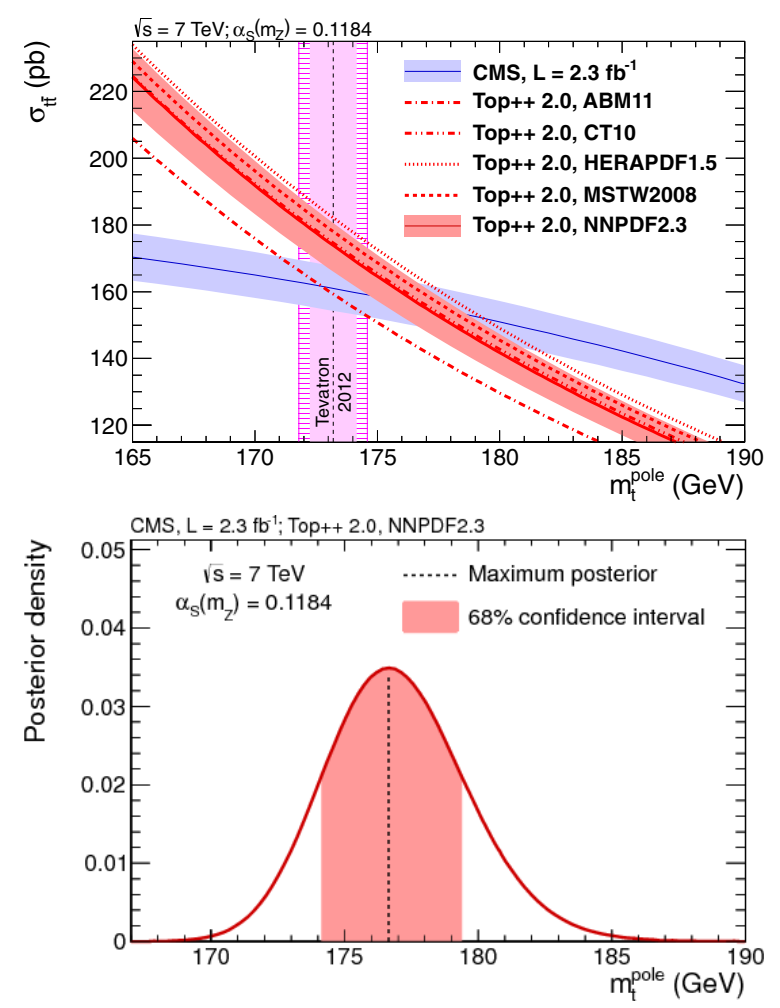

Figure 11. Upper plot: dependence of the measured and theoretical cross sections used in [29] on the top quark pole mass. The respective uncertainty bands and the result of the combinations of direct $\mathrm{M}_{\text {top }}$ measurements from the Tevatron collider as obtained in [30] are also shown.

Lower plot: the posterior probability $P\left(\mathrm{~m}_{t}\right)$. The $\mathrm{m}_{t}^{\text {pole }}$ value corresponding to the maximum of the function is taken as the measured value.

combination of measurements obtained at both the colliders has been finalized by the ATLAS, CDF, CMS and D0 Collaborations. In performing any of these combinations the correlations among uncertainties for different results are properly taken into account.

The most recent combined result has been obtained by the Tevatron Collaborations, CDF and D0 [31], and represents the best $\mathrm{M}_{\text {top }}$ measurement in the world so far, with a relative precision $\approx 0.37 \%: \mathrm{M}_{\text {top }}=174.34 \pm 0.64 \mathrm{GeV}$. The combination includes the best results from both the experiments in each measurement channel, with contributions also from the Run I of the Tevatron at $\sqrt{s}=1.8 \mathrm{TeV}$. The greatest weight on the final number comes, obviously, from the results in the lepton + jets channel and from the D0 measurement [18] presented in Sec. 4.2 in particular. CDF also updated its own combination separately, using all the measurements obtained with the full Run II statistics [32], while the last internal update by D0 has been performed in 2011 with no more than $5.4 \mathrm{fb}^{-1}$ of data [11].

As it concerns the LHC Collaborations, the best result is represented by the last individual CMS combination [19] ${ }^{1}$, where the lepton + jets measurement described

\footnotetext{
${ }^{1}$ Just after ISMD 2014 this result has been updated again to include the all-hadronic measurement [22], with a further improvement in the precision [33].
}

in Sec. 4.2 has been combined with older measurements performed with 2010 and 2011 data at $\sqrt{s}=7 \mathrm{TeV}$, for a total integrated luminosity of about $25 \mathrm{fb}^{-1}$. The combined measurement gives $\mathrm{M}_{\text {top }}=172.22 \pm 0.73 \mathrm{GeV}$, i.e. $\mathrm{a} \approx 0.43 \%$ relative uncertainty. Both the last LHC (ATLAS + CMS) and ATLAS only combinations date back to September 2013 [34] and have been performed with $\sqrt{s}=7 \mathrm{TeV}$ data only, resulting therefore in lower precisions.

The very first $\mathrm{M}_{\text {top }}$ World Average obtained by the four Tevatron and LHC experiments [35] includes the best measurements available at the time in each channel, obtained by the experiments during the Run II of the Tevatron (for CDF and D0, but the most recent updates presented in this proceeding are excluded) and with the data collected during the 2011 Run of the LHC by ATLAS and CMS. The average is $\mathrm{M}_{\text {top }}=173.34 \pm 0.76 \mathrm{GeV}$, with a precision $\approx 0.44 \%$. This result improved by $13 \%$ the best combinations existing at the time from a single collider and by $28 \%$ the best individual measurement used as an input in the combination, as can be appreciated by the summary reported in Fig. 12.

\section{Systematic Uncertainties}

The uncertainties affecting all the recent measurements of the top quark mass presented in this proceeding are dominated by systematic contributions. An example of a systematic uncertainties summary table, taken from [35], is shown in Fig. 13. The previously leading contribution due to the jet energy scale has been greatly reduced since the in situ calibration technique has been applied for the first time by the CDF Collaboration in 2006 [36] as it becomes statistical in nature. By now the largest uncertainties are tipically related to the modeling of simulated events in Monte Carlo generators and to residual JES effects. Taking the World Average [35] as an example, the two sets of sources contribute by a $30 \%$ and $16 \%$ (in quadrature) respectively to the total $0.76 \mathrm{GeV}$ uncertainty.

A great effort has been performed by all the collaborations in order to find a common way to classify the sources of uncertainty, to study the possible correlations and to avoid double countings or, on the contrary, to neglect any possible effect. Common studies and efforts are particularly relevant when combinations are performed including measurements from different channels, experiments or even colliders like in the recent World Average, and tests are performed to check the stability of results with respect to changes in the assumed correlations.

\section{Conclusions}

Since the discovery of the top quark in 1995, many measurements of its mass, which represents a fundamental parameter of the Standard Model, have been performed in all possible final states.

The CDF and D0 Collaborations at the Fermilab Tevatron collider, where the top quark was observed for the first 


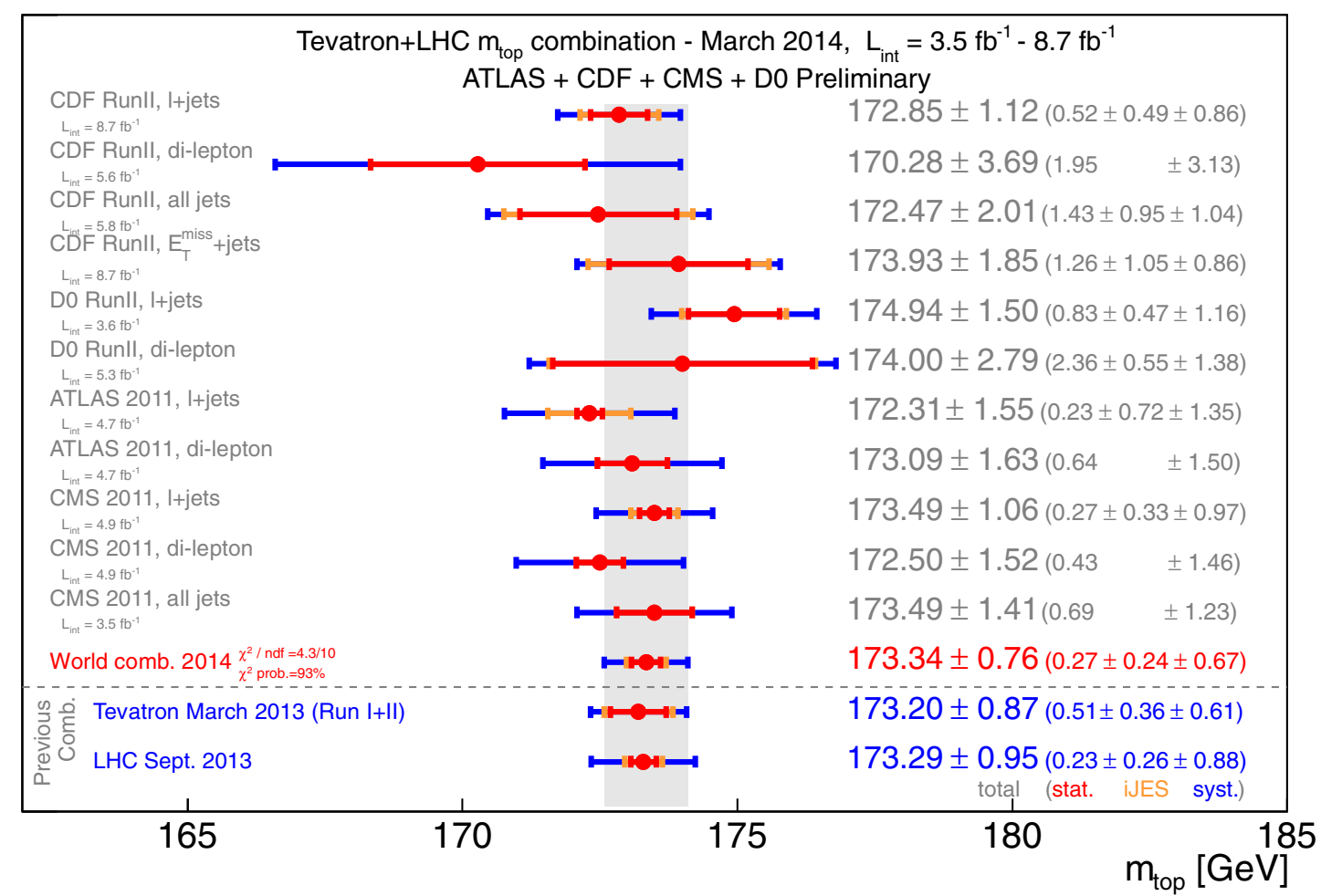

Figure 12. Input measurements to the first $M_{\text {top }}$ World Average [35] and result of their combination. The individual Tevatron and LHC combined values existing at the time are also reported.

\begin{tabular}{|c|c|c|c|c|c|c|c|c|c|c|c|c|}
\hline \multirow{2}{*}{ Uncertainty } & \multicolumn{11}{|c|}{ Input measurements and uncertainties in $\mathrm{GeV}$} & \multirow{2}{*}{$\begin{array}{r}\text { World } \\
\text { Combination }\end{array}$} \\
\hline & \multicolumn{4}{|c|}{$\mathrm{CDF}$} & \multicolumn{2}{|c|}{ D0 } & \multicolumn{2}{|c|}{ ATLAS } & \multicolumn{3}{|c|}{ CMS } & \\
\hline$m_{\text {top }}$ & 172.85 & 170.28 & 172.47 & $\frac{1}{173.93}$ & 174.94 & 174.00 & 172.31 & 173.09 & 173.49 & 172.50 & 173.49 & 173.34 \\
\hline iJES & 0.49 & n.a. & 0.95 & 1.05 & 0.47 & 0.55 & 0.72 & n.a. & 0.33 & n.a. & n.a. & 0.24 \\
\hline stdJES & 0.53 & 2.99 & 0.45 & 0.44 & 0.63 & 0.56 & 0.70 & 0.89 & 0.24 & 0.78 & 0.78 & 0.20 \\
\hline flavourJES & 0.09 & 0.14 & 0.03 & 0.10 & 0.26 & 0.40 & 0.36 & 0.02 & 0.11 & 0.58 & 0.58 & 0.12 \\
\hline $\operatorname{Rad}$ & 0.06 & 0.22 & 0.10 & 0.28 & 0.26 & 0.30 & 0.45 & 0.37 & 0.30 & 0.58 & 0.33 & 0.21 \\
\hline $\mathrm{CR}$ & 0.21 & 0.51 & 0.32 & 0.28 & 0.28 & 0.55 & 0.32 & 0.29 & 0.54 & 0.13 & 0.15 & 0.31 \\
\hline PDF & 0.08 & 0.31 & 0.19 & 0.16 & 0.21 & 0.30 & 0.17 & 0.12 & 0.07 & 0.09 & 0.06 & 0.09 \\
\hline DetMod & $<0.01$ & $<0.01$ & $<0.01$ & $<0.01$ & 0.36 & 0.50 & 0.23 & 0.22 & 0.24 & 0.18 & 0.28 & 0.10 \\
\hline$b$-tag & 0.03 & n.e. & 0.10 & n.e. & 0.10 & $<0.01$ & 0.81 & 0.46 & 0.12 & 0.09 & 0.06 & 0.11 \\
\hline LepPt & 0.03 & 0.27 & n.a. & n.a. & 0.18 & 0.35 & 0.04 & 0.12 & 0.02 & 0.14 & n.a. & 0.02 \\
\hline Total Syst & 0.99 & 3.13 & 1.41 & 1.36 & 1.25 & 1.49 & 1.53 & 1.50 & 1.03 & 1.46 & 1.23 & 0.71 \\
\hline Total & 1.12 & 3.69 & 2.01 & 1.85 & 1.50 & 2.79 & 1.55 & 1.63 & 1.06 & 1.52 & 1.41 & 0.76 \\
\hline
\end{tabular}

Figure 13. Summary of uncertainties on the measured top quark mass, taken from the first World Average [35]. In each row the corresponding uncertainty for the all input measurements ("n.a." stands for "not applicable", "n.e." for "not evaluated") and, in the last column, for the combination, are listed. The first two sections below the "Stat" row, group together the contributions related to JES and modelling of the $t \bar{t}$ signal, respectively, while in the third one other sources related e.g. to modelling of detector, analysis method etc. are included.

time and that was shut down in September 2011, are finalizing the $\mathrm{M}_{\text {top }}$ measurements, one of main goals of their physics program. The full available data samples amounts to about $10 \mathrm{fb}^{-1}$. The precision reached by individual results and in their combinations is by far beyond the goal planned for the Run II (2001-2011), and represents a very important legacy of the Tevatron.
Since 2010, when the CERN LHC started its activity, also the ATLAS and CMS Collaborations have been performing their own measurements. They are currently analyzing the data collected in 2011 and 2012, waiting for the Run II of the LHC that should start in 2015. The amount of collected data (about $25 \mathrm{fb}^{-1}$ overall), made possible by the large $t \bar{t}$ production cross section and the high istan- 
taneous luminosity of the collider, allowed to reach very small statistic uncertainties and therefore results with very good precision in a short time.

In this proceeding a selected review of the most recent and relevant results regarding $\mathrm{M}_{\text {top }}$, obtained by the four CERN and Fermilab experiments, have been presented, together with their many combinations, including the very first World Average performed in March 2014. The continuous improvements in the data analysis techniques and the understanding and calibration of detectors, made possible to achieve precisions well below $1 \%$ on the measured values, with absolute uncertainties smaller than $1 \mathrm{GeV}$. A good agreement, within the respective uncertainties, exists among all measurements and with their combinations too. The current $\mathrm{M}_{\text {top }}$ World Average, yet not including many of the results presented here, is

$$
\mathrm{M}_{\mathrm{top}}=173.34 \pm 0.76 \mathrm{GeV}
$$

\section{References}

[1] CDF Collaboration, Phys. Rev. Lett. 74, 2626 (1995).

[2] D0 Collaboration, Phys. Rev. Lett. 74, 2632 (1995).

[3] ATLAS Collaboration, Phys. Lett. B 716, 1 (2012), CMS Collaboration, JHEP 06, 081 (2013).

[4] The Gfitter Group, M. Baak et al., The global electroweak fit at NNLO and prospects for the $L H C$ and ILC, arXiv:1407.3792 [hep-ph]. Updates available from http://cern.ch/gfitter

[5] CDF Collaboration, Phys. Rev. D 71, 052003 (2005).

[6] D0 Collaboration, Nucl. Instrum. Methods A 565, 463 (2006).

[7] CDF and D0 Collaborations, Phys. Rev. D 89, 072001 (2014) and references therein.

[8] ATLAS Collaboration, JINST 3, S08003 (2008).

[9] CMS Collaboration, JINST 3, S08004 (2008).

[10] CMS and ATLAS Collaborations, Combination of ATLAS and CMS top quark pair cross section measurements in the e $\mu$ final state using protonproton collisions at $\sqrt{s}=8 \mathrm{TeV}$,

ATLAS conference note ATLAS-CONF-2014-054 (2014),

CMS Physics Analysis Summary TOP-14-016 (2014), and references therein.

[11] www-cdf.fnal.gov/physics/new/top/public_mass.html, www-d0.fnal.gov/Run2Physics/top, https://twiki.cern.ch/twiki/bin/view/AtlasPublic/TopPublicResults, https://twiki.cern.ch/twiki/bin/view/CMSPublic/PhysicsResultsTOP.

[12] ATLAS Collaboration, Measurement of the Top Quark Mass in Dileptonic Top
Quark Pair Decays with $\sqrt{s}=7$ TeV ATLAS Data, conference note ATLAS-CONF-2013-077 (2013).

[13] CMS Collaboration,

Eur. Phys. J. C72 (2012) 2202.

[14] CDF Collaboration,

Top Quark Mass Measurement in the Dilepton Channel Using the Full CDF Data Set, conference note 11072 (2014).

[15] D0 Collaboration,

Phys. Rev. Lett. 107, 082004 (2011).

[16] D0 Collaboration, Phys. Rev. D 86, 051103(R) (2012).

[17] D0 Collaboration, Phys. Rev. D 84, 032004 (2011).

[18] D0 Collaboration, Phys. Rev. Lett. 113, 032002 (2014).

[19] CMS Collaboration, Measurement of the top-quark mass in $t \bar{t}$ events with lepton+jets final states in pp collisions at $\sqrt{s}=8 \mathrm{TeV}$, Physics Analysis Summary TOP-14-001 (2014).

[20] ATLAS Collaboration, Measurement of the Top Quark Mass from $\sqrt{s}=7 \mathrm{TeV}$ ATLAS Data using a 3-dimensional Template Fit, conference note ATLAS-CONF-2013-046 (2013).

[21] CDF Collaboration, Phys. Rev. Lett. 109, 152003 (2012).

[22] CMS Collaboration, Measurement of the top-quark mass in $t \bar{t}$ events with all-jets final states in pp collisions at $\sqrt{s}=8 \mathrm{TeV}$, Physics Analysis Summary TOP-14-002 (2014).

[23] ATLAS Collaboration, Measurement of the top-quark mass in the fully hadronic decay channel from ATLAS data at $\sqrt{s}=7 \mathrm{TeV}$, arXiv:1409:0832 [hep-ex] (2014).

[24] CDF Collaboration, Phys. Rev. D 90 091101(R) (2014).

[25] CDF Collaboration, Phys. Rev. D 88011101 (2013).

[26] A. H. Hoang and I. W. Stewart, Nucl. Phys. Proc. Suppl. 185 (2008) 220, V. Ahrens et al., Phys. Lett. B 703 (2011) 135.

[27] A. Buckley et al., Phys. Rept. 504 (2011) 145.

[28] M. C. Smith and S.S. Willenbrock, Phys. Rev. Lett. 79 (1997) 3825.

[29] CMS Collaboration, Phys. Lett. B 728 (2014) 496, Phys. Lett. B 738 (2014) 526 (Corrigendum).

[30] CDF and D0 Collaborations, Phys. Rev. D 86 (2012) 092003.

[31] CDF and D0 Collaborations, Combination of $C D F$ and DO results on the mass of the top quark using up to $9.7 \mathrm{fb}^{-1}$ at the Tevatron, arXiv:1407.2682 [hep-ex].

[32] CDF Collaboration, Final combination of the CDF results on top-quark mass, conference note 11080 (2014). 
[33] CMS Collaboration,

Combination of the CMS top-quark mass measurements from Run 1 of the $\mathrm{LHC}$,

Physics Analysis Summary TOP-14-015 (2014).

[34] ATLAS and CMS Collaborations,

Combination of ATLAS and CMS results on the mass of the top-quark using up to $4.9 \mathrm{fb}^{-1}$ of $\sqrt{\mathrm{s}}=7 \mathrm{TeV} \mathrm{LHC}$ data,

ATLAS conference note ATLAS-CONF-2013-102
(2013),

CMS Physics Analysis Summary TOP-13-005 (2013).

[35] ATLAS, CDF, CMS and D0 Collaborations,

First combination of Tevatron and LHC measurements of the top-quark mass,

arXiv:1403.4427 [hep-ex] (2014).

[36] CDF Collaboration,

Phys. Rev. D 73, 032003 (2006). 